\title{
Article
}

\section{A randomised feasibility study using an acupuncture protocol to the Achilles tendon in Achilles tendinopathy}

Kishmishian, Berj, Richards, James and Selfe, James

Available at http://clok.uclan.ac.uk/24398/

Kishmishian, Berj, Richards, James ORCID: 0000-0002-4004-3115 and Selfe, James (2019) A randomised feasibility study using an acupuncture protocol to the Achilles tendon in Achilles tendinopathy. Physiotherapy Practice and Research, 40 (1). pp. 59-67. ISSN 2213-0683

It is advisable to refer to the publisher's version if you intend to cite from the work. http://dx.doi.org/10.3233/PPR-180126

For more information about UCLan's research in this area go to http://www.uclan.ac.uk/researchgroups/ and search for <name of research Group>.

For information about Research generally at UCLan please go to http://www.uclan.ac.uk/research/

All outputs in CLoK are protected by Intellectual Property Rights law, including Copyright law. Copyright, IPR and Moral Rights for the works on this site are retained by the individual authors and/or other copyright owners. Terms and conditions for use of this material are defined in the policies page.

\section{CLoK}

Central Lancashire online Knowledge www.clok.uclan.ac.uk

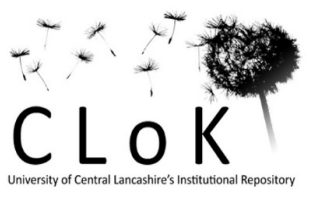




\section{A randomised feasibility study using an acupuncture protocol to}

4 Berj Kishmishian,

5 Allied Health Research Unit,

6 University of Central Lancashire,

7 Preston.

8 UK

9

10 Professor Jim Richards,

11 Allied Health Research Unit,

12 University of Central Lancashire,

13 Preston.

14 UK

15

16 Professor James Selfe,

17 Department of Health Professions,

18 Manchester Metropolitan University,

19 Manchester.

20 UK

Correspondence to

23 Berj Kishmishian,

24 Allied Health Research Unit,

25 School of Health Sciences,

26 University of Central Lancashire,

27 Preston.

28 PR1 2HE

29 UK

30 BKishmishian@uclan.ac.uk

Word Count 2847 


\section{Abstract}

2 Background: The prevalence of Achilles tendinopathy is greatest in activities including middle

3 and long distance running, tennis, badminton, volleyball, and its incidence is increasing.

4 However, currently no gold standard treatment for Achilles tendinopathy exists, although

5 eccentric exercises are commonly recommended.

6 Purpose: This study aimed to investigate the changes in clinical scores when administering a)

7 acupuncture and b) sham acupuncture to the Achilles tendon in patients diagnosed with Achilles

8 tendinopathy who did not respond to modified eccentric exercises.

9 Methods: Twenty-two patients were randomised and received either acupuncture or the control sham acupuncture treatment. VISA-A, NPRS, EQ-5D and GRC were recorded before treatment at week 0 , then at week 2, week 4 with a final follow-up review at week 12.

Main Results: Acupuncture resulted in significant differences between groups and time points in VISA-A, NPRS, EQ-5D and GRC. The Acupuncture group reached the minimum clinically important difference (MCID) threshold for important difference, when compared to sham acupuncture. The difference between treatments would suggest a beneficial response following the use of acupuncture to the Achilles tendon in Achilles tendinopathy.

17 Principal Conclusions: The overall findings suggest the use of a standardised acupuncture protocol to the Achilles tendon is a viable treatment alternative, which could be used as a second line treatment in patients diagnosed with Achilles tendinopathy who did not respond to eccentric exercises. However, in view of the small sample size, the results of this feasibility study should be viewed with caution. 


\section{Introduction}

The incidence of Achilles tendon injuries is increasing as more individuals regularly exercise both recreationally, and within sports [1,2]. The prevalence of Achilles tendinopathy is greatest in activities involving endurance running [3-6]. Injuries are also seen in sedentary individuals, with $65 \%$ of patients diagnosed with Achilles tendinopathy having no link to sport or physical activity [7].

The mid-portion of the Achilles tendon is the most commonly injured site accounting for 55$65 \%$ of all Achilles tendon injuries [3]. Due to the quality and methodological shortcomings in studies [8], there is still no gold standard treatment for Achilles tendinopathy [9]. At present the greatest amount of evidence supports eccentric exercise as a first line treatment option [1012], however, this is not always successful. Acupuncture is one of the best known complementary therapy treatments, and is widely used for musculoskeletal disorders and has been shown to offer pain relief [13]. Pain relief may be related to spinal acupuncture mechanisms through the effects of counter stimulation, or supraspinal acupuncture mechanisms through diffuse noxious inhibitory control (DNIC), and the stimulation of the descending antinocicepetive pathway and the limbic regions of the brain [14].

To date only one study by Zhang et al. [15] has explored the use of acupuncture in the treatment of Achilles tendinopathy. They reported an increase of 25.8 after 16 weeks and 28.4 after 24 weeks from baseline was found in the Victorian Institute of Sports Assessment - Achilles (VISA-A) for the acupuncture group, with a corresponding decrease of 3.2 at rest in the Visual Analogue Scale (VAS). These results suggested significant functional improvements and decreases in pain, which were greater following the use of acupuncture compared to eccentric exercise. However, when considering clinical outcome measures associated with Achilles 
tendinopathy treatments, the Numerical Pain Rating Scale (NPRS) [16], Global Rate of Change (GRC) and Euroqol-5D (EQ-5D) may also assist in identifying change in pain and health status. However, these outcome measures have yet to be validated for use on the Achilles tendon.

The aim of this randomised feasibility study was to investigate the changes in clinical scores when administering a) acupuncture and b) sham acupuncture to participants diagnosed with Achilles tendinopathy who were non-responders to a standardised eccentric loading programme.

\section{Method}

One hundred and fifty-two patients with Achilles tendon pain were referred to physiotherapy mainly through the GP service. Sixty-three patients were excluded due to; presence of insertional Achilles tendinopathy (24), naturally resolving symptoms (7), did not attend (7), medical co-morbidities (5), lumbar origin (5), other tendinopathies (3), declined intervention (3), ruptures (2), ankle OA (2), plantar fasciitis (2), unable to attend (1), referred for further investigation (1) and previous Achilles rupture (1). Of the eighty-nine eligible patients who consented, eighty-four patients (45 male, 39 female) were recruited, 80 patients (41 male, 39 female) completed the modified eccentric exercise phase (conservative treatment including the modified eccentric loading protocol).

The modified eccentric exercise protocol used in this study was adapted from Alfredson et al. [17]. Participants performed two types of eccentric exercises, with knees straight and with knees flexed, with a pain threshold of no greater than $4 / 10$ on a VAS. Both exercises were performed bilaterally or unilaterally, depending on the level of discomfort not exceeding the 
pain threshold, using minimal concentric movement. Each exercise included up to 15 repetitions performed in 3 sets once per day.

Ethical approval was gained from NRES Committee North West - Greater Manchester South REC 12/NW/0035, the University of Central Lancashire - BuSH 067 with Research and Development approval obtained from Southport and Ormskirk NHS Trust - 2011/059/LTC. All procedures followed during the study were in accordance with the Helsinki Declaration.

All patients were provided with modified eccentric exercises (within a pain framework - pain no greater than VAS 4/10) that allowed minimal pain free concentric movement. Standard static stretching was also provided coupled with appropriate footwear advice, orthotics, and management advice. During this 6-week phase patients were seen four times, two weeks apart over a 6-week period. The non-responders to modified eccentric exercises and standard treatment were invited to be randomised into two treatment groups, a) acupuncture or b) sham acupuncture. The randomisation was performed using a computer generated random number list. Once consent had been provided for the acupuncture phase, digital weighing scales were used to measure body weight, and stature was measured using a standard tape measure against a wall, whilst bioelectric impedance analysis was used to measure percentage body fat. The non-responders then attended three acupuncture/sham acupuncture treatments which were performed at weekly intervals, week 0 , week 1 and week 2 . Figure 1 shows the Achilles tendinopathy patient treatment and assessment flowchart.

$$
\text { 'Insert Figure } 1 \text { here' }
$$

The Acupuncture treatment was provided by a member of the Acupuncture Association of Chartered Physiotherapists (AACP) and followed AACP guidelines. The Acupuncture 
technique used was standardised 9-needle Achilles tendon acupuncture protocol [18], Figure 2.

101 The Achilles tendon was cleaned using alcohol wipe sterets, with participants positioned in prone lying. Nine small plastic rings covered with sterile tape were then attached to the Achilles

103 tendon to enable the Streitberger sham acupuncture needles to remain in place [19].

104 Acupuncture or sham acupuncture needles were then inserted into the Achilles tendon, with the

105 needles stimulated for 60 seconds every 5 minutes in a thrusting/twisting motion. The

106 acupuncture session ended 30 minutes after the last acupuncture or sham acupuncture needle

107 was inserted, then needles were removed.

110 All patients were assessed using the VISA-A, NPRS, GRC and EQ-5D forms. The GRC has

111 been used as an 11 point Likert scale in numerous studies [20,21] with [22] recommending the 112 use of an 11-point scale for GRC over other scales. The EQ-5D is a standardised measure of 113 health status, and is a quality of life questionnaire which comprises of 5 questions relating to 114 health, mobility, ability to self-care, ability to undertake usual activities, and anxiety and 115 depression, which have been shown to be valid and reliable [23-25]. Although, no data has

116 linked these measures with Achilles tendinopathy. When used as a secondary outcome measure, 117 these provided a more holistic assessment of Achilles tendinopathy. From the 5 health 118 dimensions measured, scores are then inserted into the EQ5D index value calculator which 119 provide an index of health. Clinical scores were measured before acupuncture and sham 120 acupuncture treatment and assessment at week 0 (session 1), before treatment on week 2 121 (session 2), before review and reassessment at week 4 (session 3) and before the final followup review at week 12 (session 4). 
126 Differences between the two groups and time points in the VISA-A, NPRS, GRC. VISA-A and

127 NPRS scores were tested for normality of distribution using the Kolmogorov-Smirnov test and

128 were found to be normally distributed and suitable for parametric statistical testing, whereas

129 the EQ-5D scores were found to be non-normatively distributed. A Repeated Measures

130 ANOVAs was used for the VISA-A and NPRS scores and a Mann-Whitney U test was used

131 for the EQ-5D scores. In addition to the Repeated Measures ANOVAs the effect sizes were

132 also calculated, and $\mathrm{p}$ values $<0.05$ were regarded as significant. The differences between

133 groups and time points were further explored using mixed methods ANOVAs. All data analysis

134 was performed using SPSS version 21 (Chicago, IL, USA).

136 To determine clinical importance two factors were considered; the minimum clinically 137 important change (MCIC) from baseline of pre-treatment to a certain time point in a primary 138 endpoint of a treatment, and the minimum clinically important difference (MCID) between 139 treatment groups [26]. The clinically important thresholds for the outcome measures were based 140 on previous research with 16 points for the VISA-A [27], 2 points for the average pain for the

141 NPRS [28] and an index value of 0.074 for the EQ-5D [29]. The GRC was dichotomised [30,31]

142 into responders and non-responders, the rate of success was expressed as patients who achieved

$143+3,+4$ and +5 points, from an 11 point GRC scale (ranging from -5 much worse to +5 much 144 better).

\section{Results}

147 Twenty-two patients who were non-responders consented to enter the study. These consistent 148 of 8 male and 14 female patients aged between 35 and 72 with a mean age of 51.8 years, height 
1491.70 meters (0.1), weight $89.3 \mathrm{~kg}$ (14.1), percentage body fat $34.5 \%$ (7.0\%) and had a BMI of $150 \quad 30.2(3.1)$

152 Differences between the two groups confirmed a significant difference $(\mathrm{p}<0.001)$ between 153 acupuncture and sham acupuncture groups. The total change in acupuncture and sham 154 acupuncture EQ-5D from baseline to the final follow-up session in week 12 was 0.16 and 0.01

155 respectively. Overall acupuncture showed a greater increase in EQ-5D score in comparison to 156 sham acupuncture by 0.15 . The Repeated Measures ANOVA show a significant mean increase 157 in VISA-A score following both acupuncture and sham acupuncture. Additionally, a significant 158 decrease in NPRS score was seen in the acupuncture group, whilst no significant difference 159 was seen in the sham acupuncture group, table 1.

163 Further pairwise comparisons were conducted for the significant main effects, table 2 . The

164 acupuncture group showed a significant difference between all weeks/sessions $(\mathrm{p}<0.001$ to $165 \mathrm{p}<0.002$ ), with the exception of week 4 to week 12, where treatment effects stabilised. For sham acupuncture, a significant increase between the baseline in week 0 to week $4(p=0.002)$, and to the final follow-up session in week $12(\mathrm{p}=0.016)$. For the NPRS scores the acupuncture group showed significant differences between the baseline at week 0 and week 2 ( $\mathrm{p}<0.001)$, and

169 between weeks 4 and $12(\mathrm{p}<0.003)$, table 2 . The largest change occurred between baseline in 170 week 0 and week 4 , with a decrease of 2.91 points, although this then increased slightly by 0.36 171 points at the final follow up session in week 12. 
175 The mixed methods ANOVA showed no significant difference in NPRS and VISA-A scores

176

177

178

179

180

181

182

183

184

185

186

187

188

189

190

191

192

\section{Discussion}

194 Acupuncture resulted in a statistically and clinically significant increase in VISA-A scores from

195 baseline to session 4 by 27 points. When compared from baseline, the use of acupuncture to the

196 Achilles tendon in Achilles tendinopathy exceeded the 16 point threshold for VISA-A MCIC and MCID at 12 weeks. The greatest increase in VISA-A was noted in session 3 and 4, at week

1984 and week 12 respectively, following the acupuncture treatment. This suggests that 
improvement seen following the final acupuncture treatment in week 2 was maintained for the follow-up periods.

201

202 Only one previous study by Zhang et al. [15] has explored the use of acupuncture measured 203 pain and function using the VISA-A. This reported a significant increase in at 8 weeks by 22.1 204 points, at 16 weeks by 25.8 points and at 24 weeks by 28.4 points which supports the findings of this study. However, Zhang et al. [15] used 4 acupuncture needles into a painful area in the Achilles tendon, which could not be kept consistent between treatments or participants; as the painful area can increase or decrease in size between treatment and participants.

The increase in VISA-A score following acupuncture is similar to that reported by Tumilty et al. [27] and Rompe et al. [32]. They reported significant increases from baseline to 18.5 points at the week 4, increasing to 32.4 points at 12 weeks and an increased VISA-A scores in Achilles tendinopathy following eccentric loading and Extra Corporeal Shock Wave Therapy (ESWT) respectively. Eccentric loading increased by 25 points at the 4 month follow-up compared to 214 the ESWT group increase by 20.1 points. Rompe et al. [33] also reported increased VISA-A scores in Achilles tendinopathy following both eccentric loading only and eccentric loading coupled with ESWT. The eccentric loading group increased by 22.7 points at 4 months; whereas the combined eccentric with the ESWT group increased by 36.3 points.

Acupuncture resulted in a 2.5 point NPRS decrease in pain compared to a 0.27 point decrease following sham acupuncture. Therefore, the standardised acupuncture protocol to the Achilles tendon in the Achilles tendinopathy RCT met the MCIC and MCID 2 point threshold for the decrease in pain using the NPRS outcome measure. The greatest decrease in pain occurred at week 4. Similar to the results of the VISA-A, if a greater number of treatments had been 
administered, this may have resulted in greater reductions in pain. Similarly, Rompe et al. [33] reported a significant decrease in NPRS in Achilles tendinopathy following both eccentric loading only and eccentric loading coupled with ESWT. The eccentric loading group decreased by 3.1 points at 4 months, whereas the combined eccentric with ESWT group decreased by 4.4 points. Conversely, Tumilty et al. [27] reported a non-significant decrease in NPRS at 4 weeks when using low-level laser therapy (LLLT) to the Achilles tendon, which suggests acupuncture to the Achilles tendon may be more useful than LLLT in Achilles tendinopathy.

The overall difference in average Achilles tendon pain over a one week period using NPRS between acupuncture and sham acupuncture, resulted in a significant difference of -1.55 points (-2 points). No significant difference were seen in the NPRS following sham acupuncture, despite an initial decrease from baseline to session 2 by 1 point. Interestingly by session 4 at week 12, pain returned to baseline values, suggesting sham acupuncture is ineffective in reducing pain. The initial reduction in pain is likely to be related to mixed mechanisms of a placebo response, and the limbic touch response [34,35], and suggests any reduction in pain occurring from sham acupuncture may be short lived.

In addition, the standardised acupuncture protocol to the Achilles tendon in the Achilles

243 for the improvement in health [29]. This was supported by the positive and statistically 244 significant VISA-A and NPRS data that show all values change in the same direction, 245 suggesting improvement and an effective treatment. Furthermore, GRC data demonstrated that $24673 \%$ of patients in the acupuncture group responded to treatment. Therefore, if a patient has 247 less pain, this could result in an increase in function and activities of daily living. 
250 The potential physiological mechanisms behind the effects found in this study could be related 251 to the local and segmental effects of acupuncture reported by Tian et al. [36]. The clinical 252 improvements noted in the acupuncture group, may be related to the local increase in blood 253 flow and oxygenation, through pro-inflammatory effects and the mechanism of axon reflexes 254 and the inhibition of the sympathetic nervous system [37,38]. The stimulation of A-Delta and 255 C-afferent fibres would release vasoactive and pro-inflammatory neuropeptides such as 256 calcitonin gene-related peptide (CGRP), substance P, neurokinin and opioids. This would result 257 in peripheral vasodilation in to the Achilles tendon, which is mainly mediated by CGRP [39]. The release of growth factors such as vascular endothelial growth factor following acupuncture could promote an increased vascular response following acupuncture and assist in Achilles 260 tendon healing, by the local increase in fibroblasts and tenocytes which result in cellular 261 proliferation and collagen synthesis [40]. The functional improvement seen in the VISA-A could suggest tissue healing may have occurred, which could enable the structure and function of the Achilles tendon to return to its pre-injury status [41].

The local reduction in pain may also be related to the spinal acupuncture mechanisms through the effects of counter stimulation [14]. The supraspinal acupuncture mechanisms of pain relief through the DNIC, could account for a short-term pain relief following treatment in the few patients that experienced this. The reduction in pain may be related to the stimulation of the 269 descending antinocicepetive pathway and the limbic regions of the brain. This would result in 270 the release of betaendorphins, adrenocorticotropic hormone and cortisone. Studies using fMRI $271[36,42]$ have reported that a strong DeQi stimulation resulted in significant deactiviations in the 272 brain, indicating a mechanism for pain relief. 
274 The standardised Achilles tendon acupuncture protocol [18] on patients with Achilles 275 tendinopathy, is suggested to primarily stimulate the Achilles tendon locally, causing local pro276 inflammatory healing and pain relieving effects. The use of acupuncture could also activate all 277 three mechanisms of acupuncture analgesia, locally, segmentally/spinally and supraspinally 278 [39].

$280 \quad$ Strengths and Limitations

281 No dropouts were recorded in the 22 patients randomised into the acupuncture or sham 282 acupuncture treatment groups which increases the confidence in the results by reducing the bias 283 that can be introduced through dropouts. The non-specific effects of acupuncture and sham 284 acupuncture [43] were controlled as the same protocol, practitioner, patient-therapist interaction 285 resulted in equal empathy and communication to all patients between groups. However, this was a single blinded randomised feasibility study where only the participant was blinded, and where the principle investigator in this study was also the acupuncturist and physiotherapist, which could introduce bias.

290 Although both needling techniques were uncomfortable, the intensity during a 60 second stimulation of an acupuncture is stronger than the stimulation of a sham needle. However, as no patient had experienced penetrative acupuncture to the Achilles tendon, this was not felt to 293 affect blinding. Improvements in pain may be, in part related to the 4 day relative rest phase 294 between treatment and by following a pain framework of not exceeding VAS 4/10 for general 295 activities. However, as the majority of patients had rested and offloaded unsuccessfully prior to 296 attending the study and followed the pain framework in the modified eccentric exercise phase 297 of the study design, therefore it is unlikely that this could account for the magnitude of the 298 differences between groups. 
300 Due to sample population group not performing hopping actions in daily activity, this was 301 considered to introduce an unnecessary risk. Therefore, in this study the Hop element 302 comprising of 10 points was omitted from the VISA-A questionnaire. Although this would 303 reduce the risk of Achilles tendon rupture, the VISA-A was developed for sporting athletes 304 rather than for sedentary patients who do not take part in regular vigorous exercise. This 305 possibly distorted the baseline and final outcome measure values, which should be considered 306 when making future comparisons. Furthermore, as patients in the acupuncture group returned 307 to their previous levels functional activities, and were able to self-manage their symptoms on 308 discharge, this may suggest that acupuncture is a beneficial treatment alternative and why the 309 relatively low final score of 60 on the VISA-A was achieved following acupuncture treatment.

\section{$311 \quad$ Recommendation for clinical practice}

312 The positive effect of the 6 week modified eccentric loading phase in this study, coupled with 313 standard physiotherapy management advice (within a pain framework - pain no greater than 314 VAS 4/10) on Achilles tendinopathy, is an imperative first line treatment, which is supported 315 by previous studies [10-12]. The results of this randomised feasibility study suggest a minimum 316 of 3 weekly acupuncture sessions may be required to achieve of positive outcome in non317 responders to the recommended first line treatment for Achilles tendinopathy. During 318 acupuncture treatments, the recommended 4 days of relative rest between acupuncture sessions 319 is advocated, before gradually progressing loading, exercise and function, within a pain 320 framework.

\section{Conclusion}


323 This randomised feasibility study has shown statistically and clinically significant improvement 324 in VISA-A, NPRS, EQ-5D and GRC following acupuncture to the Achilles tendon in patients 325 with Achilles tendinopathy. Acupuncture reached the MCIC and MCID threshold for important 326 change for all clinical scores. Whereas sham acupuncture failed to meet any MCIC or MCID 327 threshold for important change in clinical scores. This study shows that acupuncture can have 328 a positive effect in $73 \%$ of non-responders to the first line treatment of Achilles tendinopathy. 329 This suggests that the use of a standardised acupuncture protocol to the Achilles tendon is a 330 viable treatment alternative and an effective second line treatment in patients diagnosed with 331 Achilles tendinopathy who are non-responsive to eccentric exercise 
333 [1] Kader D. Achilles tendinopathy: some aspects of basic science and clinical management. Br J 334 Sports Med. 2002;36:239-249.

335 [2] Maffulli N, Sharma P, Luscombe KL. Achilles tendinopathy: aetiology and management. J $336 \quad$ R Soc Med. 2004;97:472-6.

337 [3] Järvinen T, Kannus P, Mafulli N, Khan K. Achilles tendon disorders: Etiology and 338 epidemiology. Foot Ankle Clin. 2005;10:255-266.

339 [4] Clement DB, Taunton JE, Smart GW. Achilles tendinitis and peritendinitis: etiology and treatment. Am J Sports Med. 1984;12:179-184.

341 [5] Johansson C. Injuries in enlite orienteers. Am J Sports Med. 1986;14:410-415.

342 [6] Lysholm J, Wiklander J. Injuries in runners. Am J Sports Med. 1987;15:168-171.

343 [7] de Jonge S, van den Berg C, de Vos RJ, van der Heide HJ, Weir A, Verhaar JA, Bierma344 Zeinstra SM, Tol JL. Incidence of midportion Achilles tendinopathy in the general 345 population. Br J Sports Med. 2011; 45:1026-1028.

346 [8] Alfredson H, Cook J. A treatment algorithm for managing Achilles tendinopathy: new 347 treatment options. Br. J. Sports Med. 2007;41:211-6.

348 [9] Lopez R, Jung, H. Achilles Tendinosis: Treatment Options. Clin Orthop Surg. 2015;7:1-7.

349 [10] Maffulli N, Longo U, Loppini M, Denaro V. Current treatment options for tendinopathy. $350 \quad$ Expert Opin Pharmacother. 2010;11:2177-86.

351 [11] Zwiers R, Wiegerinck JI, van Dijk CN. Treatment of midportion Achilles tendinopathy: an 352 evidence-based overview. Knee Surg Sport Traumatol Arthrosc. 2014;1-9.

353 [12] Scott L, Munteanu SE, Menz H.B. Effectiveness of Orthotic Devices in the Treatment of 354 Achilles Tendinopathy: A Systematic Review. Sport Med. 2015;45:95-110.

355 [13] Sandkühler J. Models and mechanisms of hyperalgesia and allodynia. Physiol Rev. 356 2009;89:707-758. 
357 [14] Le Bars D, Villanueva L, Bouhassira D, Willer JC. Diffuse noxious inhibitory controls 358 (DNIC) in animals and in man. Patol fiziol Eksp Ter. 1992;55-65.

359 [15] Zhang B, Zhong L, Xu S, Jiang H, Shen J. Acupuncture for chronic achilles tendnopathy: A $360 \quad$ randomized controlled study. Chin J Integr Med. 2012;19:900-904.

361 [16] Breivik EK, Björnsson GA, Skovlund E. A comparison of pain rating scales by sampling 362 from clinical trial data. Clin J Pain. 2000;16:22-8.

363 [17] Alfredson H, Pietila T, Jonsson P, Lorentzon R. Heavy-Load Eccentric Calf Muscle 364 Training For the Treatment of Chronic Achilles. The American Journal of Sports $365 \quad$ Medicine. 1998;26:360-366.

366 [18] Kishmishian B, Selfe J, Richards J. A historical review of acupuncture the the Achilles tendon and the development of a standardized protocol for its use. J Acupunct Assoc Chart Physiother. 2012;69-78.

369 [19] Streitberger K, Kleinhenz J. Introducing a placebo needle into acupuncture research. Lancet. $1998 ; 352: 364-5$.

371 [20] Pengel L, Refshauge K, Maher C. Responsiveness of pain, disability, and physical 372 impairment outcomes in patients with low back pain. Spine. 2004;29:879-883.

373 [21] Ferreira M, Ferreira P, Latimer J, Herbert R, Maher C, Refshauge K. Relationship between 374 spinal stiffness and outcome in patients with chronic low back pain. Man Ther. 2009;14:61-67.

376 [22] Kamper S, Maher C, Mackay G. Global Rating of Change scales. A Review of Strengths and Weaknesses and Considerations for Design. J Man Manip Ther. 2009;17:163-170.

378 [23] The Chartered Society of Physiotherapy. Quality Assurance Standards. CSP. London: 2014.

379 [24] Golicki D, Niewada M, Karlinska A, Buczek J, Kobayshi A, Janssen M, Pickard A. 380 Comparing responsiveness of the EQ-5D-5L, EQ-5D-3L and EQ VAS in stroke patients. $381 \quad$ Qual Life Res. 2014;24:1555-1563. 
382 [25] Janssen M, Pickard A, Golicki D, Gudex C, Niewada M, Scalone L, Swinburn P, Busschbach

383 J. Measurement properties of the EQ-5D-5L compared to the EQ-5D-3L across eight $384 \quad$ patient groups: A multi-country study. Qual Life Res. 2013;22:1717-1727.

385 [26] Togo K, Matsuoka N, Hashigaki S, Imai K, Moriya T. Clinically important effects in new 386 drug development. Drug Inf J. 2011;45:805-10.

387 [27] Tumilty S, McDonough S, Hurley D, Baxter G. Clinical effectiveness of low-level laser 388 therapy as an adjunct to eccentric exercise for the treatment of Achilles' tendinopathy: A 389 randomized controlled trial. Arch Phys Med Rehabil. 2012;93:733-739.

390 [28] Farrar J, Pritchett Y, Robinson M, Prakash A, Chappell A. The Clinical Importance of 391 Changes in the 0 to 10 Numeric Rating Scale for Worst, Least, and Average Pain Intensity. $392 \quad$ J Pain. 2010;11:109-118.

393 [29] Walters SJ, Brazier JE. Comparison of the minimally important difference for two health 394 state utility measures: EQ-5D and SF-6D. Qual Life Res. 2005;14:1523-1532.

395 [30] van der Roer N, Ostelo R, Bekkering G, van Tulder M, de Vet H. Minimal clinically 396 important change for pain intensity, functional status, and general health status in patients 397 with nonspecific low back pain. Spine. 2006;31:578-582.

398 [31] Collins N, Crossely K, Beller E, Darnell R, McPoil T, Vicenzino B. Foot orthoses and 399 physiotherapy in the treatment of patellofemoral pain syndrome: randomised clinical trial. $400 \quad$ Br J Sports Med. 2009;43:169-171.

401 [32] Rompe J, Nafe B, Furia J, Maffulli N. Eccentric loading, shock-wave treatment, or a wait402 and-see policy for tendinopathy of the main body of tendo Achillis: a randomized $403 \quad$ controlled trial. Am J Sports Med. 2007;35:374-83.

404 [33] Rompe J, Furia J, Maffulli N. Eccentric loading versus eccentric loading plus shock-wave 405 treatment for midportion achilles tendinopathy: a randomized controlled trial. Am J Sports Med. 2009;37:463-70. 
407 [34] Lundeberg T. To be or not to be: the needling sensation (de qi) in acupuncture. Acupunct. $408 \quad$ Med. 2013; 31:129-31.

409 [35] Lund I, Näslund J, Lundeberg T. Minimal acupuncture is not a valid placebo control in 410 randomised controlled trials of acupuncture: a physiologist's perspective. Chin Med. $411 \quad 2009 ; 4: 1-9$.

412 [36] Tian, D, Xiong J, Pan Q, Liu F, Wang L, Xu S, Huang G, Wang W. De Qi, a Threshold of 413 the Stimulus Intensity, Elicits the Specific Response of Acupoints and Intrinsic Change 414 of Human Brain to Acupuncture. Evid Based Complement Alternat Med. 2014;1-11.

415 [37] Carlsson C. 2002. Acupuncture mechanisms for clinically relevant long-term effects416 reconsideration and a hypothesis. Acupunct. Med. 2002;20:82-99.

417 [38] Shinbara H, Okubo M, Kimura K, Mizunuma K, Sumiya E. Contributions of nitric oxide 418 and prostaglandins to the local increase in muscle blood flow following manual $419 \quad$ acupuncture in rats. Acupunct. Med. 2015;33:65-71.

420 [39] Lundeberg T. Acupuncture mechanisms in tissue healing:contribution of NO and CGRP. Acupunct. Med. 2013;7-8.

422 [40] Docheva D, Muller SA, Majewski M, Evans CH. Biologics for tendon repair. Adv Drug 423 Deliv Rev. 2015;84:222-239.

424 [41] Sereysky JB, Flatow EL, Andarawis-Puri N. Musculoskeletal regeneration and its 425 implications for the treatment of tendinopathy. Int J Exp Pathol. 2013;94:293-303.

426 [42] Hui KK, Marina O, Liu J, Rosen BR, Kwong KK. Acupuncture, the limbic system, and the 427 anticorrelated networks of the brain. Auton Neurosci. 2010;157:81-90.

428 [43] Langevin HM, Wayne PM, Macpherson H, Schnyer R, Milley RM, Napadow V, Lao 1, Park J, Harris R, Cohen M, Sherman K, Haramati A, Hammerschlag R. Paradoxes in 430 acupuncture research: strategies for moving forward. Evid Based Complement Alternat $431 \quad$ Med. 2011;1:1-11. 
432 Table 1: Mean change from baseline and standard deviation (sd) for VISA-A and NPRS

433 and Repeated Measures ANOVA main effects with effect $\operatorname{size}\left(\boldsymbol{\eta}_{\boldsymbol{p}}^{2}\right)$

\begin{tabular}{|c|c|c|c|c|}
\hline \multirow{2}{*}{} & \multicolumn{2}{|c|}{ VISA-A } & \multicolumn{2}{c|}{ NPRS } \\
\cline { 2 - 5 } & Acupuncture & Sham & Acupuncture & Sham \\
\cline { 2 - 5 } & Mean (sd) & Mean (sd) & Mean (sd) & Mean (sd) \\
\hline Week 0 & $33.73(15.49)$ & $40.55(19.12)$ & $5.54(1.86)$ & $4.54(1.37)$ \\
\hline Week 2 & $47.91(16.51)$ & $50.09(22.92)$ & $3.91(1.70)$ & $3.54(2.07)$ \\
\hline Week 4 & $60.36(16.63)$ & $50.27(19.45)$ & $2.64(1.96)$ & $3.36(1.63)$ \\
\hline Week 12 & $60.73(19.54)$ & $51.82(22.22)$ & $3.00(2.19)$ & $4.27(2.19)$ \\
\hline p-value & $<\mathbf{0 . 0 0 1}$ & $\mathbf{0 . 0 3 0}$ & $<\mathbf{0 . 0 0 1}$ & 0.201 \\
\hline effect size & 0.670 & 0.254 & 0.558 & 0.141 \\
\hline
\end{tabular}

434 
Table 2: Repeated Measures ANOVA Pairwise comparisons for significant main effects

for NPRS and VISA-A for Acupuncture and Sham groups.

\begin{tabular}{|c|c|c|c|}
\hline \multicolumn{4}{|c|}{ Repeated Measures ANOVA } \\
\hline & $\begin{array}{c}\text { Mean } \\
\text { Difference }\end{array}$ & p-value & $\begin{array}{l}\text { Confidence Intervals of } \\
\text { the Differences }(95 \%)\end{array}$ \\
\hline \multicolumn{4}{|c|}{ NPRS - Acupuncture Group } \\
\hline Week $0-2$ & $1.64^{*}$ & $<0.001$ & 0.95 to 2.33 \\
\hline Week $0-4$ & $2.91^{*}$ & $<0.001$ & 1.98 to 3.83 \\
\hline Week $0-12$ & $2.54^{*}$ & 0.003 & 1.06 to 4.03 \\
\hline Week $2-4$ & $1.27^{*}$ & 0.003 & 0.53 to 2.01 \\
\hline Week $2-12$ & 0.91 & 0.148 & -0.38 to 2.20 \\
\hline Week $4-12$ & -0.36 & 0.596 & -1.84 to 1.12 \\
\hline \multicolumn{4}{|c|}{ VISA-A - Acupuncture Group } \\
\hline Week $0-2$ & $-14.18^{*}$ & $<0.002$ & -21.90 to -6.46 \\
\hline Week $0-4$ & $-26.64^{*}$ & $<0.001$ & -36.16 to -17.11 \\
\hline Week $0-12$ & $-27.00^{*}$ & $<0.001$ & -38.89 to -15.11 \\
\hline Week $2-4$ & $-12.46^{*}$ & $<0.001$ & -18.30 to -6.61 \\
\hline Week $2-12$ & $-12.82^{*}$ & 0.020 & -23.18 to -2.46 \\
\hline Week $4-12$ & -0.36 & 0.908 & -7.19 to 6.46 \\
\hline \multicolumn{4}{|c|}{ VISA-A - Sham Group } \\
\hline Week $0-2$ & -9.54 & 0.061 & -19.65 to 0.56 \\
\hline Week $0-4$ & $-9.73^{*}$ & 0.016 & -17.23 to -2.22 \\
\hline Week $0-12$ & $-11.27^{*}$ & 0.002 & -17.13 to -5.41 \\
\hline Week 2-4 & -0.18 & 0.969 & -10.40 to 10.03 \\
\hline Week $2-12$ & -1.73 & 0.720 & -12.18 to 8.73 \\
\hline Week 4-12 & -1.54 & 0.657 & -9.06 to 5.97 \\
\hline
\end{tabular}


443 Table 3: Mixed methods ANOVA Pairwise comparisons between the Sham and

444 Acupuncture treatment groups.

\begin{tabular}{|c|c|c|c|}
\hline \multicolumn{4}{|c|}{ Mixed Methods ANOVA } \\
\hline & $\begin{array}{c}\text { Mean } \\
\text { Difference }\end{array}$ & p-value & $\begin{array}{c}\text { Confidence Intervals of } \\
\text { the Differences (95\%) }\end{array}$ \\
\hline \multicolumn{3}{|c|}{ NPRS } \\
\hline $\begin{array}{c}\text { Sham vs } \\
\text { Acupuncture }\end{array}$ & $-1.545^{*}$ & $<\mathbf{0 . 0 0 1}$ & -2.366 to -.725 \\
\hline \multicolumn{3}{|c|}{ VISA-A } \\
\hline $\begin{array}{c}\text { Sham vs } \\
\text { Acupuncture }\end{array}$ & $12.424^{*}$ & $<\mathbf{0 . 0 0 1}$ & 5.838 to 19.011 \\
\hline
\end{tabular}

445

446 

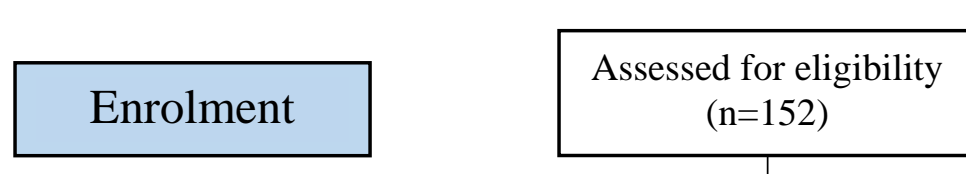

$$
450
$$

451
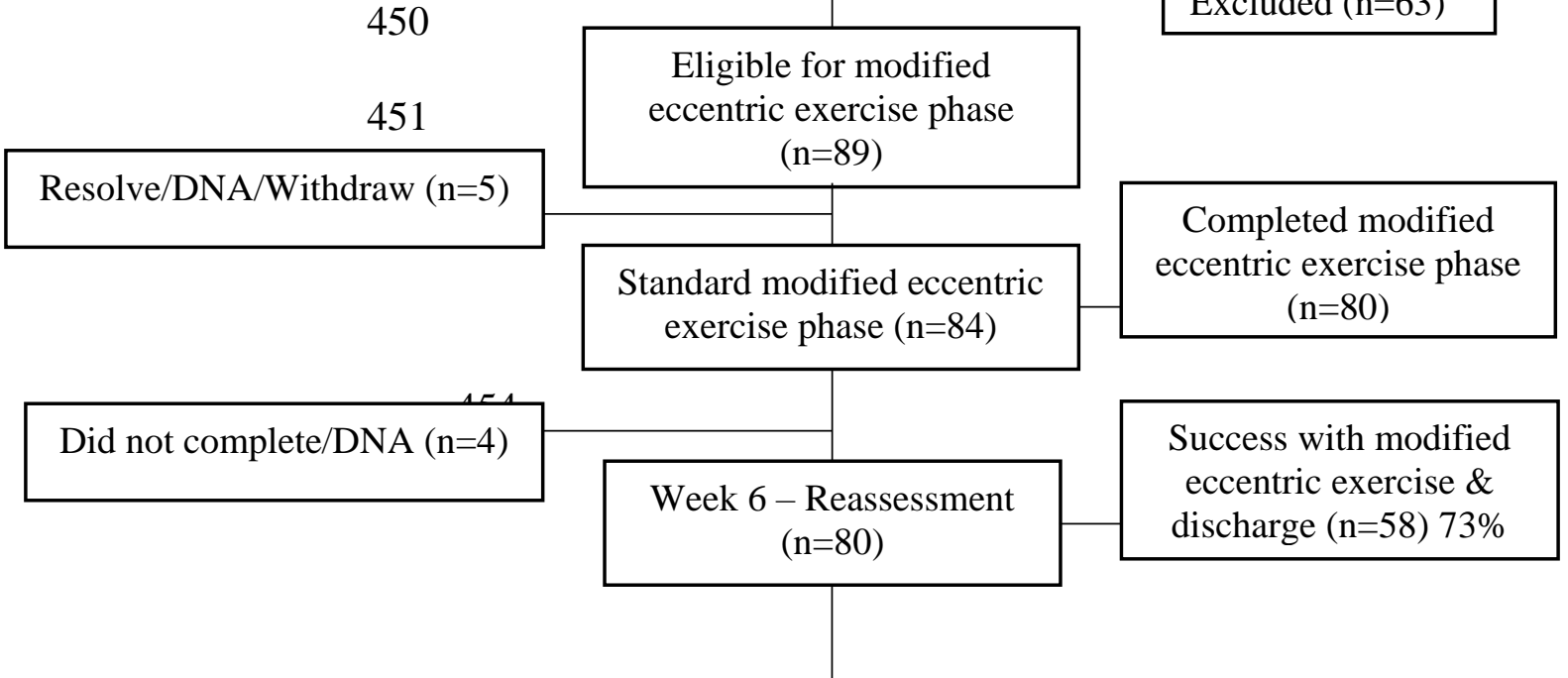

Excluded $(\mathrm{n}=63)$

Acupuncture phase - randomised non-responders to standard modified eccentric exercises $(\mathrm{n}=22) 27 \%$
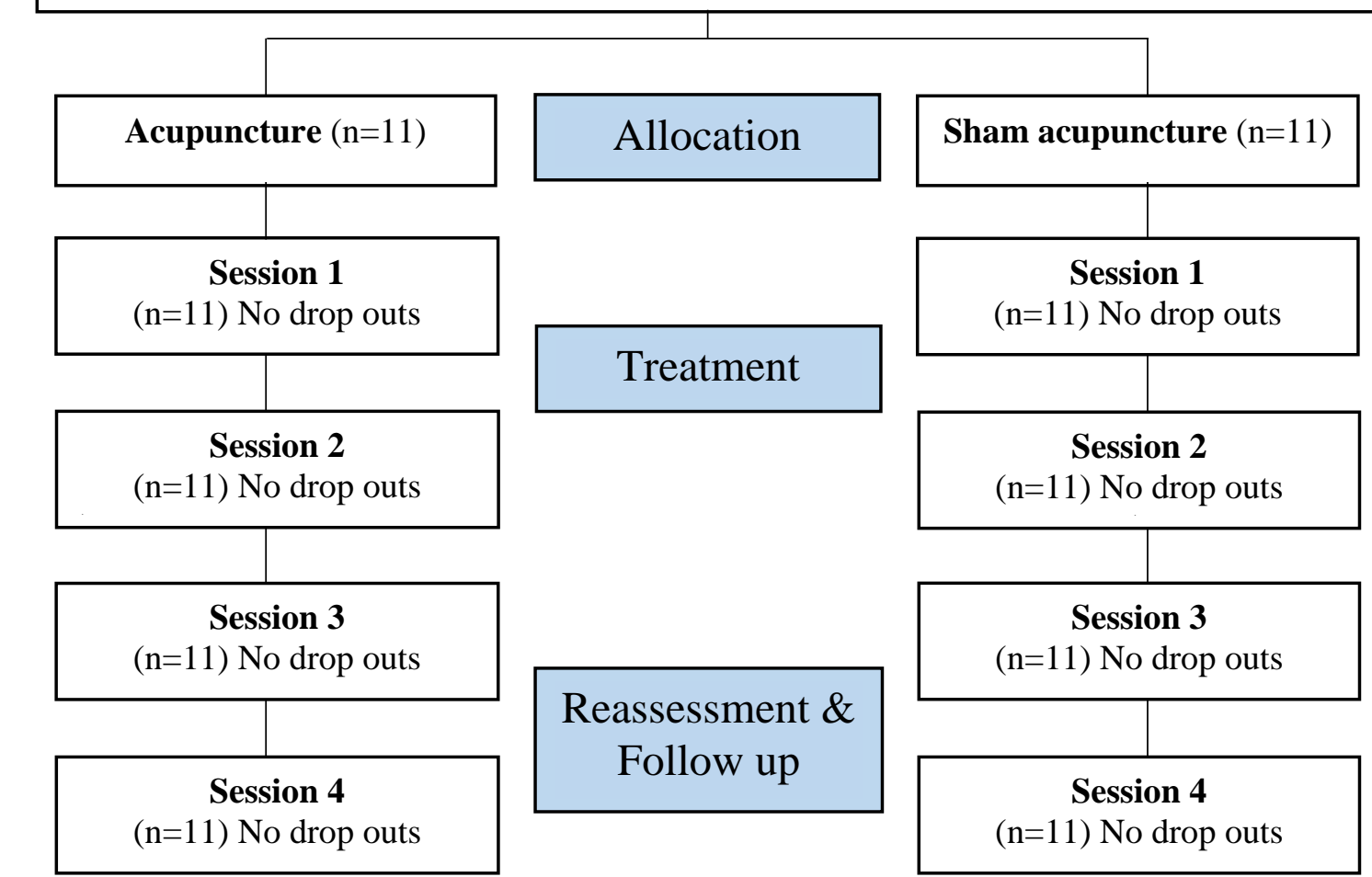

Session 1 (week 0) \& Session 2 (week 2)=Physiotherapy assessment, completion of clinical scores prior to treatment, and NIRS/TI physiological measurements before, during and after acupuncture/sham treatment. 
471 Figure 2: The standardised 9-needle Achilles tendon acupuncture protocol inserted into

472 the Achilles tendon (Kishmishian et al, 2012).

473

474

475

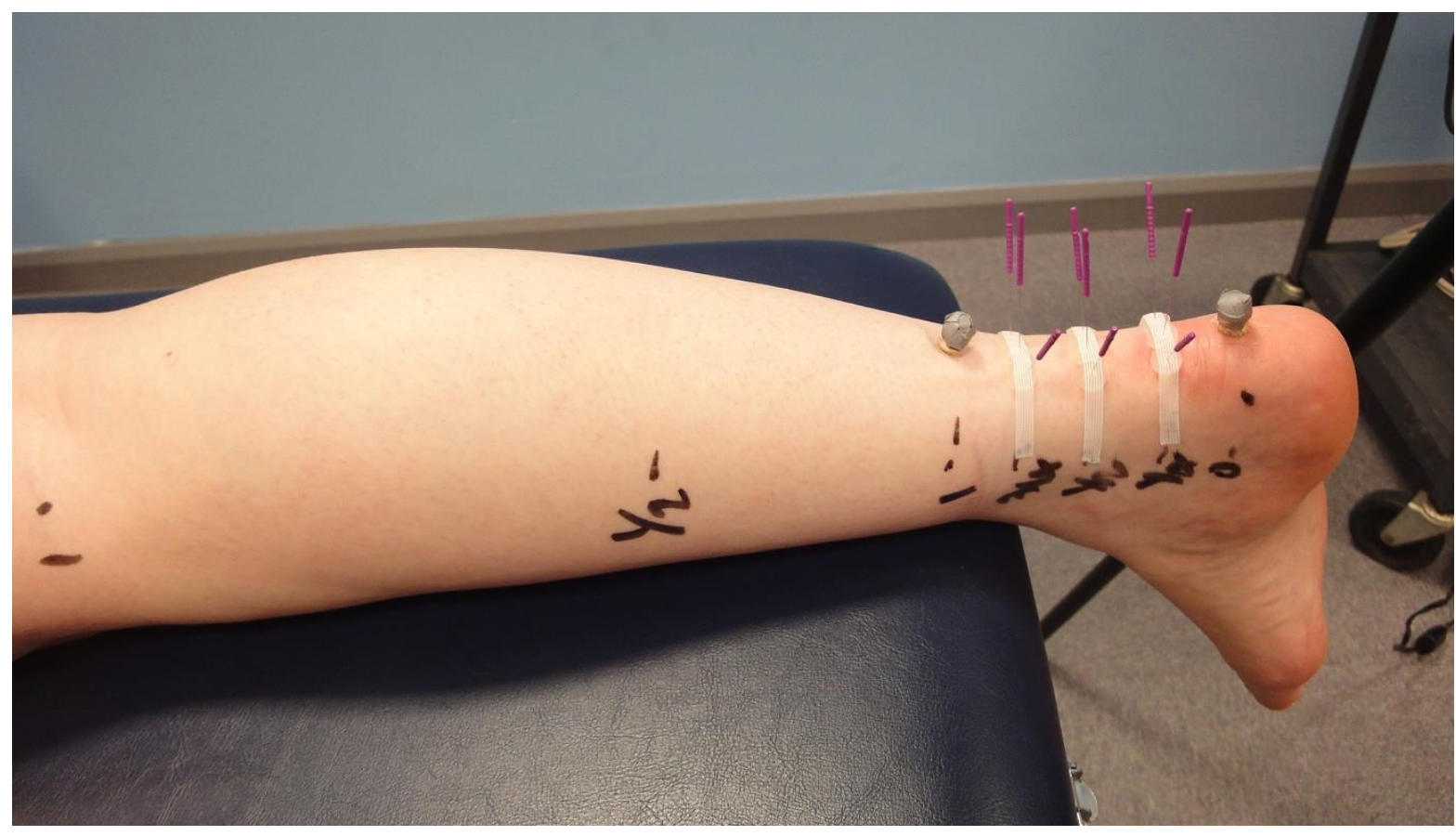

476

477

478

479

480

481

482

483

484

485

486 